\title{
Incontri
}

Anno 35, 2020 / Fascicolo 2 / p. 39-53 - www.rivista-incontri.nl - http://doi.org/10.18352/incontri.10350 (c) The author(s) - Content is licensed under a Creative Commons Attribution 3.0 Unported License - Publisher: Werkgroep Italië Studies, supported by Utrecht University Library Open Access Journals

\section{Quando lo 'scandalo' diventa provocazione Il postsecolare e il teatro iconoclasta di Romeo Castellucci}

\section{Monica Jansen}

Introduzione: i limiti tra provocazione e scandalo

Lo spettacolo Sul concetto di volto nel Figlio di Dio di Romeo Castellucci ha destato polemiche fin dal suo approdo in Francia nel 2011 al Festival di Avignone. Se il pubblico in prima istanza consisteva di singoli indignati, si è poi venuto a identificare con gruppi di cattolici integralisti manifestatisi per la prima volta a Parigi nel 2011 con l'occupazione del palcoscenico del Théâtre de la Ville. ${ }^{1}$ Anche al teatro Parenti a Milano, dove è stato rappresentato nel gennaio del 2012, lo spettacolo ha incontrato resistenze che questa volta hanno in parte coinvolto anche la direttrice del teatro, André Ruth Shammah, in quanto ebrea.

A sostegno del regista non si sono mossi soltanto intellettuali laici e rappresentanti dei lavoratori dello spettacolo, ma anche figure istituzionali religiose. A testimonianza del diffrangersi delle posizioni cattoliche, in occasione delle rappresentazioni a Bologna in programma al Teatro Comunale Testoni a Casalecchio di Reno il 17 e il 18 febbraio 2012, ${ }^{2}$ La Repubblica di Bologna pubblica due pareri contrari, uno firmato dal vescovo di Anversa, l'altro dal cardinale Carlo Caffarra. Il primo dei due, da 'spettatore credente', si dice profondamente commosso dal messaggio spirituale della pièce, a cui ha assistito al teatro De Singel, e distingue chiaramente la propria competenza di 'pastore' da quella dell'artista: 'Porre una domanda lasciando allo stesso tempo la risposta aperta: questa è la forza di un artista'. Il secondo, che parla invece facendosi propria la voce di 'cittadini' e 'credenti', afferma che questi sarebbero 'sdegnati e addolorati', come cittadini 'nel vedere che l'esercizio della libertà espressiva non conosce più neppure i limiti del rispetto dell'altro', e come credenti 'nel vedere inserito il Volto Santo [...] in uno spettacolo indegno, offensivo, e obiettivamente blasfemo e sacrilego'. Il cardinale incita poi i 'buoni fedeli di Casalecchio' a 'reagire in modo fermo e composto' allo spettacolo. ${ }^{3}$ Mentre le

\footnotetext{
${ }^{1} \mathrm{~F}$. Di Mercurio, 'Le scandale des violences esthétiques de Romeo Castellucci', in: Les chantiers de la création. Revue pluridisciplinaire en Lettres, Langues, Arts et Civilisations, 6 (2013), http: / /journals. openedition.org/ Icc/500 (14 maggio 2020).

2 G. Marino, 'La Societas Raffaello Sanzio al Teatro Testoni', BolognaCult, 17 febbraio 2012, https://www.bolognacult.it/2012/02/16/sul-concetto-di-volto-nel-figlio-di-dio/ (14 maggio 2020).

3 J. Bonny, 'In quel volto la forza del dubbio', La Repubblica Bologna, 17 febbraio 2012, https://bologna.repubblica.it/cronaca/2012/02/17/news/in_quel_volto_la_forza_del_dubbio_la_recensione _del_vescovo_belga-30044203/; C. Card. Caffarra, 'Sdegnati e addolorati', La Repubblica Bologna, 16 febbraio
} 
proteste finora passate in rassegna replicano le tesi e antitesi tra chi intende la spiritualità al di fuori dei confini dottrinali della religione e chi invece la intende in un senso assoluto ed esclusivamente cristiano, l'episodio di Le Mans del 2018, dove la prefettura della Sarthe ha posto il divieto sull'impiego di bambini sulla scena, sposta l'attenzione dai manifestanti cattolici verso l'istanza che dovrebbe garantire la libertà artistica.

Questa serie di incidenti ha costretto il regista a legittimare in pubblico la sua arte e le sue scelte che i suoi detrattori hanno tacciato di blasfemia e sacrilegio o anche di "cristianofobia". Facendo uso degli spazi che i media gli hanno concesso, Castellucci ha difeso la sua idea estetica di arte come 'scandalo', e al contempo ha affermato che il suo è un teatro ispirato ai valori cristiani e rispettoso della dimensione etica della religione. Anzi, il suo sarebbe stato un tentativo di sottrarre la figura di Cristo al monopolio simbolico della destra cattolica. ${ }^{4}$ Questo contributo intende vagliare i confini tra provocazione e 'scandalo' con un'opera artistica che adopera un cosciente montaggio della trasgressione estetica iconoclasta e il codice simbolico dell'iconologia cristiana. Mentre la libertà di espressione viene tutelata dallo stato secolare democratico, questo diritto "naturale" non coincide necessariamente con il rispetto per le minoranze religiose richiesto in una società postsecolare e multietnica. Le polemiche intorno alla pièce di Castellucci e le dichiarazioni di poetica del regista fanno vedere i limiti entro i quali si configura sia un'arte postsecolare che non perda la sua capacità di fare 'scandalo' sia un pubblico postsecolare aperto alla messa in questione delle verità assolute secolari o religiose.

Il postsecolare: la 'denaturalizzazione' del secolare e la messa in opera dell'iconoclash L'insieme delle turbolenze intorno a Sul volto ci offre, nel complesso, l'opportunità di indagare il conflitto tra la libertà creativa dell'artista e il rispetto per la religione all'interno del dibattito sulla società postsecolare. La cosiddetta "svolta" postsecolare, databile intorno al Duemila, può essere identificata con la personalizzazione di pratiche religiose nella sfera privata che comportano una distinzione tra religione e spiritualità, ${ }^{5}$ da un lato, e dall'altro con il discusso "ritorno" della religione nella sfera pubblica. ${ }^{6}$ Una produttiva sintesi del dibattito socio-teologico e politico sulla società postsecolare è quella proposta da Umut Parmaksız, che propone di definire il postsecolare come la 'denaturalizzazione' postreligiosa del secolare, che con il suo potere critico offre gli strumenti teorici per 'denaturalizzare' l'ordine normativo sia del secolare che del religioso. In questa visione la religione e il secolare sono concepite come categorie non essenzialiste e relative a certi comportamenti, conoscenze e sensibilità che trovano la loro prassi nell'ambito delle vicende umane, a cui ogni contestazione dell'autorità naturale di una delle due deve rispondere. Il secolare è quindi tutt'altro che un campo

2012, https://bologna.repubblica.it/cronaca/2012/02/17/news/sdegnati_e_addolorati_la_lettera_di_carlo_ caffarra-30042506/ (14 maggio 2020).

${ }^{4}$ D. Semenowicz, The theatre of Romeo Castellucci and Societas Raffaello Sanzio: From icon to iconoclasm, from word to image, from symbol to allegory, New York, Palgrave Macmillan, 2016, p. 163.

${ }^{5} \mathrm{Si}$ veda $\mathrm{M}$. Zonch, 'Il testimone di fede. Verità e spiritualità nella narrativa di Saviano', in: Incontri. Rivista europea di studi italiani, 32, 1 (2017), p. 52: 'Senza voler ignorare le differenze tra la riflessione, ad esempio, di Beck e quella di Pace o di Daniel Hervieu-Léger e di Luigi Berzano, si può infatti affermare che, almeno in occidente, a fronte di una progressiva perdita di terreno delle religioni tradizionali si stia assistendo a un rifiorire della vita spirituale'.

${ }^{6}$ Punto di riferimento qui è J. Habermas, 'Religion in the Public Sphere', in: European Journal of Philosophy, 1 (2006), pp. 1-25. Il filosofo tedesco sostiene che il carattere secolare dello stato sia una condizione necessaria ma non sufficiente per garantire una uguale libertà religiosa a tutti (ivi, p. 4). Oltre all'imparzialità dell'istituzione dello stato verso le comunità religiose, la partecipazione riuscita in una pratica condivisa di autodeterminazione democratica richiede anche un' ‘etica di cittadinanza' (ivi, p. 6). 
neutro, come il ritorno della religione non corrisponde necessariamente a colmare un vuoto di senso. ${ }^{7}$

Ambedue le interpretazioni sul valore spirituale e pubblico della religione possono essere riconosciute in produzioni culturali "postsecolari" che tematizzano le discrepanze tra interpretazioni secolari e religiose e che offrono scenari possibili per affrontare le tensioni generate. Ciò significa che la terza dimensione del postsecolare riguarda la religione nel dominio dell'estetica. Essendo l'arte un medium privilegiato per dare una 'forma sensazionale' alla religione, l'estetica diventa un campo ideale per analizzare in quali casi la configurazione artistica corrisponde al contesto religioso di una tradizione o di un gruppo, e quando invece viene sperimentata come un 'atto blasfemo'. Sono queste le domande poste dalla studiosa di religione Birgit Meyer che, analizzando l'atto materiale della 'fabbricazione del credere', si è anche occupata delle tensioni generate tra posizioni secolari e religiose quando l'immaginazione artistica risulta essere offensiva contro la religione. ${ }^{9}$ Ciò vale in particolar modo per quelle produzioni culturali intenzionalmente provocatorie, com'è il caso di certe opere femministe con connotazioni religiose, ${ }^{10}$ ma anche delle avanguardie storiche che consideravano l'atto blasfemo e sacrilego positivamente come rottura con la tradizione della religione. Secondo Meyer, l'arte prodotta dopo la tragedia dell'11 settembre, approccia la religione da un punto di vista postsecolare nel senso che, prendendo la religione sul serio come un fattore nella società contemporanea multiculturale, l'artista va in cerca di nuovi tipi di provocazione. ${ }^{11}$ Meyer inoltre fa propri i concetti teorizzati dal filosofo francese Bruno Latour, il cui fortunato neologismo di 'iconoclash' offre uno strumento per categorizzare quelle opere artistiche che si manifestano con un'ambivalenza irrisolvibile tra decostruzione di un'immagine sacra e ricostruzione su basi "altre". ${ }^{12}$

\section{L'iconoclash di Castellucci: un'iconofilia 'rovinosa'}

Per quanto riguarda il caso specifico dell'esperienza della religione cattolica in Italia, Enzo Pace la riassume con la formula di un 'generalizzato codice simbolico' che rende possibile la percezione di 'unità nella diversità'. ${ }^{13}$ Questa tradizione culturale di un cattolicesmo popolare per Clodagh Brook forma il 'cemento' di opere cinematografiche con un misto di insegnamento cattolico sociale e impegno, che però non si staccano dall'immaginario cristiano e quindi non corrispondono alla maggiore sfida posta dalla

\footnotetext{
7 U. Parmaksız, 'Making sense of the postsecular', in: European Journal of Social Theory, 21, 1 (2018), p. 107. ${ }^{8} \mathrm{Si}$ veda B. Meyer, Mediation and the Genesis of Presence. Towards a Material Approach to Religion, Utrecht, Faculteit Geesteswetenschappen, Universiteit Utrecht, 2012.

${ }^{9}$ Ivi, pp. 30-31.

$10 \mathrm{Si}$ veda A.-M. Korte, 'Blasphemous Feminist Art: Incarnate Politics of Identity in Postsecular Perspective', in: R. Braidotti, B. Blaagaard, T. de Graauw, \& E. Midden (a cura di), Transformations of religion and the public sphere: postsecular publics, Houndmills, Basingstoke, Hampshire: Palgrave Macmillan, 2014, pp. 22848.

${ }^{11}$ B. Meyer, 'The Dynamics of Taking Offense. Concluding Thoughts and Outlook', in: C. Kruse, B. Meyer, \& A. -M. Korte (a cura di), Taking Offense. Religion, Art, and Visual Culture in Plural Configurations, Paderborn, Wilhelm Fink, pp. 345.

12 Il filosofo si riferisce con il neologismo a 'those sites, objects, and situations where there is an ambiguity, a hesitation, an iconoclash on how to interpret image-making and image-breaking'. B. Latour, 'What is Iconoclash? Or is there a world beyond the image wars?', in: P. Weibel \& B. Latour (eds.), Iconoclash, Beyond the Image-Wars in Science, Religion and Art, Cambridge, ZKM and MIT Press, 2002, pp. 23.

${ }^{13}$ E. Pace, 'A peculiar pluralism', in: Journal of Modern Italian Studies, 12, 1(2007), p. 89.
} 
società postsecolare: quella di fare i conti con una società multi-religiosa in cui non tutti i membri condividono gli stessi valori etici. ${ }^{14}$

All'interno di questo contesto postsecolare e allo stesso tempo predominantemente cattolico, lo spettacolo Sul volto di Castellucci si inserisce nella tradizione iconologica cristiana italiana ed europea, di cui il regista si dichiara 'erede'. ${ }^{15} \mathrm{Si}$ inserisce così in quella tradizione atea e cattolica nel cinema italiano descritta da Brook, ${ }^{16}$ per cui non è da escludere un'affinità di poetica tra Castellucci e il comunista cattolico Pier Paolo Pasolini che, condannato a quattro mesi di prigione nel 1963 per vilipendio alla religione ${ }^{17}$ per il suo cortometraggio La ricotta, dichiarò ai giudici:

lo, per me, sono anticlericale (non ho mica paura a dirlo!) ma so che in me ci sono duemila anni di cristianesimo: io coi miei avi ho costruito le chiese [...] barocche: esse sono il mio patrimonio, nel contenuto e nello stile. ${ }^{18}$

Ne risulta una tensione 'ironica', caratteristica per il contesto italiano, tra la trasgressione dialettica dell'approccio iconoclasta e la riaffermazione delle regole e dei codici precostituiti. ${ }^{19}$ Tale ambivalenza interpretativa che caratterizza il riferimento alla tradizione cattolica nell'arte contemporanea italiana, può anche essere compresa nei termini di Latour, e dunque essere il risultato di un 'iconoclash'. Per quanto riguarda l'opera di Castellucci lo si può affermare in forza di alcune sue dichiarazioni di poetica, al cui centro troviamo il concetto di 'iconoclasmo' della compagnia Societas Raffaello Sanzio ${ }^{20}$ a cui sono state dedicate diverse pubblicazioni. ${ }^{21}$ Il teatro della compagnia è 'iconoclasta' nella misura in cui, come spiega Dorota Semenowicz, l'immagine per Castellucci non è solo lo strumento per costruire il linguaggio scenico, ma anche il soggetto di una riflessione filosofica, etica ed estetica. ${ }^{22}$

Per definire il ruolo dell'immagine l'autore ricorre infatti alla nozione di 'iconoclasmo' nel suo significato letterale di 'rompere l'immagine'. ${ }^{23}$ L'iconoclasmo di Castellucci agisce contro un'immagine intesa nei termini mimetici di imitazione, che,

\footnotetext{
${ }^{14}$ C. Brook, 'Post-secular identity in contemporary Italian cinema: Catholic "cement", the suppression of history and the lost Islamic other', in: Modern Italy, 22, 2 (2017), p. 207.

15 Semenowicz, The theatre of Romeo Castellucci and Societas Raffaello Sanzio, cit. p. 62.

${ }^{16}$ Brook identifica un gruppo di registi italiani tra cui Pasolini, Bellocchio e Sorrentino che si definiscono atei, agnostici o anticlericali, ma che al contempo non riescono a fare a meno dei rituali e delle icone del cattolicesimo. C. Brook, Screening Religions in Italy: Contemporary Italian Cinema and Television in the Postsecular Public Sphere, Toronto, Toronto University Press, 2019, p. 62.

17 L'accusa del Pubblico Ministero sostiene: 'tutto il film si svolge nelle sue scene, nelle sue inquadrature, nelle sue sequenze e nei commenti musicali e verbali in maniera tale che la religione cattolica risulta dileggiata e derisa nei suoi simboli e nelle sue manifestazioni più toccanti ed essenziali'. Cit. in: A. Marchesini, Citazioni pittoriche nel cinema di Pasolini: da Accattone al Decameron, Firenze, La Nuova Italia, 1994, p. xCIII. 18 Ivi, p. XCIII.

${ }^{19}$ S. Bassi, 'The Tragedy in Italy', in: The Oxford Handbook of Shakespearean Tragedy, a cura di M. Neill \& D. Schalkwyk, Oxford, Oxford UP, 2016, p. 704.

${ }^{20}$ La compagnia è stata fondata a Cesena nel 1981 dai fratelli Claudia e Romeo Castellucci insieme a Chiara Guidi e un numero di altri artisti. Nel 1990 il termine latino 'Societas' ha sostituito quello di 'Società' per denotare l'affinità e l'unione di singoli individui verso uno scopo comune. Nel 2006 Claudia Castellucci, Romeo Castellucci e Chiara Guidi hanno scelto di perseguire ognuno la propria strada artistica (E. Papalexiou \& A. Xepapadakou, 'About SRS', 2017, https://www.arch-srs.com/srs.

${ }^{21}$ A. Mango \& T. Colusso, Il teatro iconoclasta, Ravenna, Essegi, 1989; C. Castellucci, R. Castellucci \& Societas Raffaello Sanzio, Il teatro della Societas Raffaello Sanzio. Dal teatro iconoclasta alla super-icona, Milano, Ubulibri, 1992.

22 Semenowicz, The theatre of Romeo Castellucci, cit., p. 2.

23 Ibidem.
} 
priva di un'idea, si riduce a mero idolo. ${ }^{24} \mathrm{Si}$ tratta di un progetto etico-estetico, in cui l'iconoclasmo non contraddice l'immagine, ma ne decostruisce una visibilità per liberarne un'altra. In altre parole, in questa visione - che si presenta in bilico tra gli storici iconoclasti e gli iconofili, un'altra similitudine con l'iconoclash nell'interpretazione di Latour - l'iconoclasmo ambisce rompere l'icona per rendere il pubblico soggetto al suo carattere oggettivo e astorico. Non si tratta però per Castellucci di raggiungere l'essenza nel senso di divina trascendenza, ma l'operazione di montaggio introduce l'elemento della contraddizione, che crea spazio per l'esperienza emotiva dello spettatore. ${ }^{25}$

La 'terza immagine' creata dal montaggio nell'interpretazione del cineasta Sergej Eisenstein è fondamentale per Castellucci per comprendere l'esperienza emotiva, che il pubblico fa delle contraddizoni generate dal teatro iconoclasta, in termini di 'tensioni' e non tanto di 'opposizioni' tra icona e iconoclasmo. ${ }^{26}$ L'atto di rottura, fondato nel cambiamento di prospettiva, deve essere spietato per liberare le emozioni nello spettatore, e perciò il pubblico lo sperimenta come un atto di violenza. ${ }^{27}$ Dato l'aspetto dualistico dell'iconoclasmo di Castellucci, a metà strada tra destruzione e ricostruzione dell'immagine, il suo progetto artistico, da lui stesso caratterizzato come 'lotta iconoclasta', ${ }^{28}$ è anche stato analizzato nei termini di una 'metafisica rovinosa', in alternativa a una metafisica 'trascendentale' o invece 'critica' ${ }^{29} \mathrm{Ne}$ testimonia la sua pratica ostensiva di rovinamento del corpo, linguaggio, suono, immagine, che non mira a decretare l'obsolescenza del teatro come medium, ma invece a ricreare una tradizione teatrale non mimetica, rifondata nella sua materialità corporea. ${ }^{30}$ Dirigendo il suo iconoclasmo verso il dramma classico, Castellucci crea costellazioni teatrali che confrontano gli spettatori con una poetica frammentata e spesso scioccante senza offrire interpretazioni unificanti. ${ }^{31}$ Così facendo il rovinamento viene elevato ad attività centrale per la sua pratica performativa e non è riducibile unicamente a un processo inevitabile di decadenza degenerativa. È quindi lecito concludere che il rovinamento iconoclasta adoperato da Castellucci diventi un atto di 'decostruzione costruttiva' che demolisce la tradizione del teatro come mezzo per re-inventarlo diversamente. ${ }^{32}$

In questa luce acquista particolare rilievo per il regista teatrale la nozione biblica di 'rivelazione' che, riletta attraverso Nietzsche secondo il quale l'arte è uno strumento che rende possibile di liberarsi dalla verità, combina il dubbio inerente alla verità cristiana con il ruolo del teatro che è quello di disturbare la comunicazione. ${ }^{33}$ L'opportunità di scoperta o di rivelazione così creata non equivale secondo Castellucci a misticismo, a una conoscenza che viene dall'alto, ma invece appartiene alla dimensione esistenziale del tragico, in cui l'uomo si trova da solo dopo la morte degli dei. L'orizzonte della liberazione non è quello della salvazione che si trova fuori dalla vita quotidiana, ma invece la trasgressione che viene dall'interno del teatro e che nella tragedia greca fa parte del sistema. Posto in questi termini, la finzione contraffatta del teatro non offre l'esperienza

\footnotetext{
24 Ivi, p. 34.

25 Ivi, p. 35.

26 Ivi, p. 36.

27 Ibidem.

${ }^{28}$ Cit. in T. De Laet \& E. Cassiers, 'The Regenerative Ruination of Romeo Castellucci', in: Performance Research, 20, 3 (2015), p. 19.

29 Ivi, p. 21.

30 Ibidem.

31 Ivi, p. 27.

32 Ibidem.

33 Semenowicz, The theatre of Romeo Castellucci, cit., pp. 161-162.
} 
luminosa della salvezza ma il buio in cui lo spettatore deve trovare la propria luce o 'epifania'. 34

Proprio questa convinzione del tragico che trasgredisce la legge dall'interno come un 'veleno', ${ }^{35}$ ha spinto l'artista di Cesena alla definizione di un teatro dello 'scandalo', nel senso etimologico di sasso sulla strada in cui si inciampa, come egli non smette di sottolineare nelle interviste, distinguendo così il suo atto creativo di urto e riflessione da quello calcolato della provocazione. ${ }^{36}$ Si tratta in altre parole di un teatro dell' 'appello', volto a sensibilizzare lo spettatore, più che un mezzo per produrre agitazioni e indignazioni sterili. ${ }^{37}$

\section{Il volto di Cristo tra "rifiuto" e "specchio"}

Lo spettacolo Sul concetto viene collocato dalla critica nel terzo periodo dell'opera artistica di Castellucci, insieme alla produzione Purgatorio (2008) - che fa parte del trittico dantesco Divina Commedia - e viene messo in relazione allo spettacolo Genesi: From the Museum of Sleep del 1999. Le tre performance avrebbero in comune il fatto di riflettere sulla relazione tra creatore e creazione, tra Dio e la sua opera, padre e figlio. ${ }^{38}$ Sul concetto potrebbe invece anche formare un trittico teatrale sul tema del viso e del rifiuto, come suggerisce lo stesso Castellucci, insieme al precedente Il velo nero del pastore (2010) ispirato alla novella omonima di Nathaniel Hawthorne, e al successivo The Four Seasons Restaurant (2012). ${ }^{39}$

Oltre al tema della sottrazione dell'immagine a una fruizione passiva, i tre spettacoli si uniscono anche per via del comune ricorso a una scenografia che si divide da una parte nel dramma con attori, che mettono in scena un 'teatro codificato dalle arti visive' in cui 'l'iconografia pittorica trionfa', e dall'altra nel trasformarsi dello spazio scenico in uno acustico senza più 'la presenza viva degli attori', in cui si introduce un 'effetto speciale' con la 'funzione di concludere lo spettacolo con un colpo'. ${ }^{40}$ Come recita la sinossi di Sul concetto offerta nel programma dello spettacolo a Besançon nel 2015: 'Les spectacles de la Societas Raffaello Sanzio sont des formes théâtrales où l'image prend le pas sur le texte, générant des tableaux vivants, abstraits et symboliques'. ${ }^{41}$

\footnotetext{
34 Pp. 161-162, p. 157.

35 Ivi, p. 161.

36 F. Pascaud, 'Romeo Castellucci: “Au théâtre, l'interdit, c'est la réalité”', Télérama, 6 luglio 2012, https: / /www.telerama.fr/scenes/romeo-castellucci-au-theatre-l-interdit-c-est-la-realite,83898.php maggio 2020).

37 Di Mercurio, 'Le scandale des violences esthétiques de Romeo Castellucci', cit.

38 Semenowicz, The theatre of Romeo Castellucci, cit., p. 8. Sul sito 'The archive of Societas Raffaello Sanzio' si legge che 'Introducing Genesis on the theatrical stage, the Societas Raffaello Sanzio bring the sacred words to a profane, transcendental space, out of the world, forgotten by God, away from the sacredness of the divine speech and the images it conveys. In this performance the fundamental contradiction of the human condition is stressed: on the one hand generation and creation, and on the other genocide and destruction'. Lo spettacolo combina riferimenti alla storia del fraticidio di Caino e Abele con eventi storici contemporanei quali la scoperta della radioattività e il genocidio nazista dell'Olocausto. E. Papalexiou \& A. Xepapadakou, 'About SRS', 2017, https: //www.arch-srs.com/srs (14 maggio 2020).

${ }^{39}$ Intervistato in occasione dell'esordio di The Four Seasons Restaurant al Festival di Avignone, il regista spiega a Jean-François Perrier che i tre spettacoli sono uniti da 'une histoire de rejet'. 'Entretien avec Romeo Castellucci', 2012, PDF sul sito https://www.festival-avignon.com/en/shows/2012/the-four-seasonsrestaurant (14 maggio 2020).

${ }^{40}$ N. Martino, 'Carteggio Valentini/Castellucci', in: Alfabeta2, 30 novembre 2013, https: //www.alfabeta2.it/ 2013/11/30/carteggio-valentinicastellucci/ (14 maggio 2020).

41 Il PDF del programma si trova su https://www.theatre-contemporain.net/images/upload/pdf/f-9b456aca2d7eca77.pdf (14 maggio 2020).
} 
Come si è detto, l'iconografia di Castellucci è in debito con l'eredità europea del cattolicesimo, che il regista dice di recepire come un flusso continuo di forme da leggere e decostruire in quanto espressione del pensiero dell'uomo. ${ }^{42}$ Non sembra quindi esserci un conflitto tra le immagini cristiane messe in scena dall'artista e le questioni esistenziali e morali che esse sollevano, come mettono in rilievo diverse interpretazioni dettagliate della pièce che si trovano per lo più in sintonia con le dichiarazioni di Castellucci stesso sul suo spettacolo. La scena di Sul concetto è occupata da una riproduzione ingrandita del volto di Cristo ritagliato dal Salvator Mundi di Antonello da Messina, che ne lascia fuori la mano benedicente. La scelta dell'immagine è il risultato dell' 'appello' dello sguardo divino; il regista afferma di esserci 'caduto' sopra e che il quadro, da lui studiato anni prima all'Accademia di belle arti a Bologna, richiama in lui l' ecce homo' che attraverso la sua fragilità genera nell'uomo la passione. ${ }^{43}$ Inoltre, nella lettera ai giornali del 17 gennaio 2012 in replica ai cattolici integralisti intenti a impedire lo svolgimento dello spettacolo al Teatro Parenti di Milano, Castellucci sottolinea la funzione di 'specchio' del volto di Gesù, testimone del dramma umano che avviene sulla scena:

Per questo ho scelto il dipinto di Antonello: a causa dello sguardo di Gesù che è in grado di fissare direttamente negli occhi ciascuno spettatore con una dolcezza indicibile. Lo spettatore guarda lo svolgersi della scena ma è a sua volta continuamente guardato dal volto. Il Figlio dell'uomo, messo a nudo dagli uomini, mette a nudo noi, ora. ${ }^{44}$

Lo spettacolo è diviso in tre parti. Al centro della prima, che rispetta una drammaturgia realistica, si trovano un padre anziano e incontinente in mutande e il figlio, vestito per andare in ufficio. La scena si svolge dentro un appartamento borghese bianco, "immacolato' 45 e moderno. Il dialogo consiste di poche parole, distribuite su un foglio al pubblico che segue lo spettacolo, in cui il padre chiede scusa a ripetizione al figlio di non poter trattenere le sue feci, mentre quest'ultimo, premuroso, si innervosisce. ${ }^{46}$ Attraverso lo svuotamento inarrestabile del corpo del padre Castellucci suggerisce allo spettatore una similitudine con la Kenosis, il processo di 'svuotamento' di Cristo tornato sulla terra che si destituisce dalla sua divinità per integrarsi letteralmente con la sua dimensione umana. ${ }^{47}$

Dal momento in cui il figlio imprime un bacio sulle labbra del Cristo, i due personaggi lasciano il palcoscenico che si trasforma in uno spazio aperto. Nella seconda parte si vedono dei bambini che dalle loro cartelle traggono granate giocattolo per lanciarle contro l'immagine di Cristo. Per questa scena l'artista dice di essersi ispirato a una fotografia di

\footnotetext{
42 Semenowicz, The theatre of Romeo Castellucci, cit., pp. 162, 190.

43 J.-L. Perrier, 'Entretien avec Romeo Castellucci (I/II)', Rennes 2011, riprodotto nel programma dello spettacolo a Beçanson 2015, cit.

${ }^{44}$ R. Castellucci, 'Ecco perché il mio spettacolo non ha proprio nulla di osceno', La Repubblica, 17 gennaio 2012, https://milano.repubblica.it/cronaca/2012/01/17/news/ecco_perch_il_mio_spettacolo_non_ha_pro prio_nulla_di_osceno-28258142/ (14 maggio 2020). Una versione leggermente diversa, presentata come quella 'integrale', è quella di M. Marino, 'Romeo Castellucci interviene sullo spettacolo contestato a Milano', Corriere della Sera, 17 gennaio 2012, http://boblog.corrieredibologna.corriere.it/ 2012/01/17/romeo_castellucci_interviene_s/ (14 maggio 2020).

45 Programma dello spettacolo a Beçanson 2015, cit.

46 'LE PĖRE - Pardon... Je suis désolé... Je suis désolé... /LE FILS - Arrête de t'excuser. Ça m’énerve... Allez Papa. /LE FILS - C'est bon. Ça va... [...]/ LE FILS - Mais qu'est-ce qui t'est arrivé, Papa... Qu'est-ce qui va pas... Papa...Papa... Papa... Papa.../ LE PÈRE - Pardon, je suis désolé, je suis désolé. Pardonne-moi... Pardonne-moi... Pardonne-moi...' Programma dello spettacolo a Beçanson 2015, cit.

47 Perrier, cit. nel programma dello spettacolo a Beçanson 2015, cit.
} 
Diane Arbus intitolata Child with Toy Hand Grenade in Central Park, N.Y.C. 1962, esposta nel 2011 al Jeu de Paume, casualmente in coincidenza con la messa in scena dello spettacolo al Théâtre de la Ville a Parigi. ${ }^{48}$ Nella terza e ultima parte il pubblico, confrontato unicamente con il Volto, assiste a un movimento che viene da dietro l'immagine che lo imbratta di nero - 'achiropita, non per mano d'uomo' - e che lacera il dipinto, 'come una membrana', finché non appaiano le lettere illuminate 'You are my shepherd' / 'Tu sei il mio pastore' (Salmo 23 di Davide) in cui si insinua un piccolo, grigio, 'not'/ 'non'. ${ }^{49}$ Nella stessa lettera in risposta ai cattolici integralisti di Milano il regista suggerisce di interpretare il dubbio insito nel 'non' alla luce del Salmo 22 del Re Davide:

Tu non sei il mio pastore. La frase di Davide si trasforma così per un attimo nel dubbio. Tu sei o non sei il mio Pastore? Il dubbio di Gesù sulla croce - "Dio perché mi hai abbandonato?" - espresso dalle parole stesse del salmo 22 del Re Davide. Questa sospensione, questa intermittenza della frase, racchiude il nucleo della fede come dubbio, come luce, come l'incerta condizione umana. ${ }^{50}$

Il tutto si svolge con la durata di una cinquantina di minuti, in tempi volutamente estremamente dilatati. ${ }^{51}$ In questo modo viene stimolata la creatività dello spettatore e quindi anche la sua memoria dell'esperienza della performance. Dice Castellucci a Semenowicz che tutto in Sul concetto era artificiale, ma che le emozioni rimangono vere. ${ }^{52}$

La messa in opera dell' 'iconoclash' si sposta dunque dal piano dell'estetica concettuale della pièce alla ricezione emotiva da parte del pubblico, concepito da Castellucci come creatore alla pari di Dio. ${ }^{53} \mathrm{E}$ in questa dimensione della fruizione - anche se in molti dei casi si è trattato di un esplicito rifiuto a priori - che si genera il conflitto tra l'opera e i credenti. Questi ultimi, prendendo "alla lettera" l'attacco distruttivo all'immagine di Cristo, non sembrano coglierne l'aspetto "ricostruttivo". Ancora una volta, si potrebbero giustapporre le posizioni di Castellucci a quelle di Pasolini, e in particolare la difesa dall'accusa di irriverenza del primo con l'argomentazione del secondo, contro l'accusa di vilipendio all'immagine di Gesù ne La ricotta:

Non è Cristo che sbotta a ridere - è l'umile attore che interpreta il Cristo, che non è poi neanche Cristo, ma è il Cristo profanamente raffigurato dal Pontormo. ${ }^{54}$

\footnotetext{
48 A. Héliot, 'Romeo Castellucci: la pièce qui fait scandale', Le Figaro, 31 ottobre 2011, https: / /www.lefigaro.fr/theatre/2011/10/30/03003-20111030ARTFIG00226-romeo-castellucci-la-piece-quifait-scandale.php (14 maggio 2020). Castellucci vede nella posa innaturale del ragazzino magro un'immagine di vulnerabilità, un'icona della fame spirituale della nostra epoca (Semenowicz, The theatre of Romeo Castellucci, cit., p. 95).

49 Suggestiva l'interpretazione del vescovo di Anversa: 'Per me, come cristiano, il nodo della questione è questo. Il dubbio di padre e figlio non può riconoscersi nel volto sereno del Salvator Mundi. Per tale riconoscimento hanno bisogno di ritrovare il volto sofferente del Cristo crocifisso. Volto che verrà anche mostrato loro durante lo spettacolo. Le granate lanciate dai bambini contro l'immagine del Salvator Mundi sono la versione attuale del grido della folla del Venerdi Santo: “Crocifiggilo!" (Mar. 15,13). Per alcuni tempestosi minuti bombardati da rumori violenti, la sala scompare nel buio. L'immagine del Salvator Mundi è scomparsa' (Bonny, 'In quel volto la forza del dubbio', cit.).

${ }^{50}$ Castellucci, 'Ecco perché il mio spettacolo non ha proprio nulla di osceno', cit.

51 Di Mercurio, 'Le scandale des violences esthétiques de Romeo Castellucci', cit.: 'Pour l'artiste cette lenteur jusqu'à l'arrêt de l'image est en opposition radicale avec le flux ininterrompu des images proposées par les industries médiatiques qui disqualifient toute interruption, suspens et temps d'invisibilités'.

52 Semenowicz, The theatre of Romeo Castellucci, cit., p. 159.

53 Ivi, p. 157.

${ }^{54}$ Marchesini, Citazioni pittoriche nel cinema di Pasolini, cit., p. xCIII.
} 
Castellucci si difende dall'accusa di irriverenza in modo analogo, richiamandosi all'artificio artistico che segue un proprio linguaggio semiotico, non direttamente riportabile al realismo del soggetto rappresentato, definendo il lancio delle granate giocattolo sul ritratto di Cristo "un gesto innocente portato da innocenti" motivato dal salmo 88, "Dio non nascondermi il tuo Volto":

Le immagini dure e spiacevoli del lavoro appartengono alla vita, non sono una mia invenzione sadica. Certe volte il teatro utilizza, come nella tragedia greca, una tecnica antifrastica, omeopatica; una tecnica cioè che utilizza gli elementi estranei per significare l'opposto. E così, per esempio, un gesto violento vuole significare la fragilità umana e il bisogno di amore. ${ }^{55}$

\section{La blasfemia di Sul concetto}

Che lo spettacolo, dopo l'esordio a Essen nel 2010, fin dall'inizio abbia suscitato reazioni violente in Francia, potrebbe essere dovuto al fatto che il paese adotta un secolarismo di tipo radicale. ${ }^{56}$ Dopo l'occupazione del podio del Thêatre de la Ville a Parigi da parte di giovani ultracattolici appartenenti a organizzazioni religiose vicine ai lefebrvristi, ${ }^{57}$ le rappresentazioni delle due settimane seguenti si sono dovute svolgere sotto stretta sorveglianza delle forze dell'ordine. Castellucci, intervistato nel 2012 da Semenowicz sulle manifestazioni in Francia contro la sua opera, ha detto di non curarsi delle reazioni di persone che non avevano neanche visto lo spettacolo, ma di interessarsi solamente alle reazioni del pubblico in sala, consistente sia di credenti che di non credenti. ${ }^{58}$

Gli esempi offerti dalla protesta in Francia e poi in Italia fanno vedere molto bene la contesa che anima la sfera pubblica, e a cui partecipano diversi gruppi di credenti, divisi tra di loro. Il Cardinale André Vingt-Trois, arcivescovo di Parigi e Presidente della Conferenza Episcopale Francese, ha condannato la violenza adoperata dai manifestanti non essendo loro intitolati ad agire in difesa della Chiesa, ${ }^{59}$ mentre in Italia il Vaticano ha reagito con una dichiarazione in appoggio ai manifestanti determinando la produzione 'offensiva per cristiani'.60 La tipologia delle proteste rende anche possibile studiare le motivazioni di un gruppo di credenti colpiti a tal punto nella loro integrità da aver deciso di portare avanti azioni illegali contro la blasfemia, che li hanno anche esposti al rischio

55 Castellucci, 'Ecco perché il mio spettacolo non ha proprio nulla di osceno', cit.

56 T. Modood, 'Is There a Crisis of “Postsecularism” in Western Europe?', in: Transformations of Religion and the Public Sphere. Postsecular Publics, a cura di R. Braidotti, B. Blaagaard, T. de Graauw \& E. Midden, Houndmills, Basingstoke, Hampshire, Palgrave Macmillan, 2014, p. 16: 'The majority are reacting to the minority, not to the felt constraints of "secularism" and so the form of the challenge is not a religious resurgence but an ethno-religious multiculturalism - indeed, not postsecularism but secularism, or neosecularism, is one of the leading majoritarian responses, especially in France'.

57 La perturbazione della prima del 20 ottobre 2011 è stata rivendicata ufficialmente dal movimento Renouveau Français, costituito da giovani militanti che si considerano il 'groupe choc' per l'Istituto Civitas che raccoglie cattolici tradizionalisti e integralisti vicini all'estrema destra. O. Faye, A. Mestre \& C. Monnot, 'Le Renouveau français revendique la perturbation du spectacle de Romeo Castellucci', Le Monde, 24 ottobre 2011, https: //www.lemonde.fr/politique/article/2011/10/24/le-renouveau-francais-revendique-la-perturba tion-du-spectacle-de-romeo-castellucci_5981805_823448.html (14 maggio 2020).

58 Semenowicz, The theatre of Romeo Castellucci, cit., p. 163.

59 Ivi, p. 85.

60 In verità la dichiarazione firmata da Monsignor Peter Brian Wells, assessore per gli Affari Generali della Segreteria di Stato, faceva riferimento alle parole di papa Benedetto XVI, che non erano però intese come un intervento diretto della Santa Sede, ma erano piuttosto state formulate in risposta alla lettera di un prete cattolico diretta al Pontefice. Il prete, padre Giovanni Cavalcoli, postò la risposta papale subito sul sito di Riscossa Cristiana, che in quel momento guidò le proteste a Milano, da dove è stata poi diffusa ai giornalisti. C. Balme, 'The Affective Public Sphere: Romeo Castellucci's On the Concept of the Face Regarding the Son of God’, in: Tiyatro Eleștirmenliği ve Dramaturji Bölümü Dergisi / Sayı, 22, 1 (2013), pp. 108-109. 
dell'arresto, di multe e condanne alla prigione. ${ }^{61}$ La manifestazione è a sua volta diventata un medium promozionale contro la 'cristianofobia', con la diffusione di un video attraverso il sito di Renouveau Français, ${ }^{62}$ trasformando così il palcoscenico in un vero e proprio foro pubblico. ${ }^{63}$ La contesa promossa dai cattolici tradizionalisti e nazionalisti, inoltre, ha trovato il supporto di gruppi nazionalisti e neofascisti sia in Francia che in Italia. ${ }^{64}$

L'azione contro Sul concetto ha fatto parte di un'ondata di proteste guidate dall'istituto Civitas e dall'AGRIFF (l'Alleanza generale contro il razzismo e per il rispetto dell'identità francese e cristiana) sotto lo slogan di 'combattimento culturale', campagna iniziata con una petizione contro l'opera fotografica 'Piss Christ' di Andres Serrano, esposta alla collezione Lambert a Avignone dal 12 dicembre 2010 all'8 maggio 2011, e diventata più intensa nell'autunno a Parigi contro la programmazione di due pezzi teatrali ritenuti blasfemi dai cattolici oltranzisti, lo spettacolo di Castellucci a ottobre-novembre e Golgotha picnic del regista argentino Rodrigo Garcia a dicembre.

Le proteste contro Garcia erano già state preparate in anticipo, in seguito agli attacchi alla pièce degli ambienti dei cattolici tradizionalisti in Spagna. Le tre opere contestate hanno in comune il fatto di essere immediatamente riconoscibili, sin dal titolo, come opere che hanno qualcosa a che fare con il sacro, di essere poi firmate da autori non francesi e di corrispondere alla tipologia d'arte contemporanea ripudiata da Civitas in quanto non corrispondente alla verità. ${ }^{65} \mathrm{~A}$ luglio 2011, la rivista Civitas aveva pubblicato un dossier speciale intitolato 'Combat culturel', in cui si offrivano al lettore cattolico gli strumenti per poter distinguere la buona arte da quella moralmente sbagliata. In concreto, l'arte buona per Civitas, che prende in prestito i dettami di Pio XII, ${ }^{66}$ è esemplare nel senso che rappresenta comportamenti e sentimenti degni di essere imitati, istruttiva perché fa conoscere gli elementi della storia evangelica, e trascendentale perché porta a Dio. Al contrario, la pseudo-arte contemporanea è brutta, provocatoria, immorale, e a volte perfino blasfema. Ė inutile quando prende se stessa per fine, e nociva quando 'fait profession d'abaisser et asservir aux passions charnelles les puissances spirituelles de l'âme'. ${ }^{67}$

Per di più, per i cattolici integralisti di Civitas, il concetto di finzione equivale a quello di eresia. La verità assoluta e l'unicità del sacro non possono essere presentate in una finzione come una semplice possibilità, che apre così alla relatività e alla pluralità dei significati. Seguendo questa logica, il fatto che una finzione artistica si appropri della storia sacra e delle sue figure è condannabile per principio. Ciò vuol dire che ogni variazione a partire dalla storia sacra è considerata blasfema, ancor di più quando l'opera compie un 'attentato' all'immagine, come avviene letteralmente in Sul concetto quando i bambini lanciano le loro bombe contro il volto di Cristo. La raffigurazione di Cristo,

\footnotetext{
61 Ivi, p. 111.

62 S. D'Urso, 'On the Theology of Romeo Castellucci's Theatre and the Politics of the Christian "Occupation" of His Stage', in: Theatre Research International, 38, 1 (2013), pp. 34-35.

63 Balme, 'The Affective Public Sphere', cit., p. 110.

64 Ivi, pp. 108-109.

${ }^{65}$ M. Barraband \& J.-Y. Camus, 'Le combat culturel des traditionalistes catholiques. L'affaire Golgotha picnic, un coup de force?', in: ConTextes. Revue de sociologie de la littérature, 26 (2020), https://doi.org/10.4000/contextes.8733 (14 maggio 2020).

66 Il ricorso ai dettami di Pio XI e XII stabilisce un ritorno anacronistico alle pratiche dell'Indice descritte in M. Brera, Novecento all'Indice. Gabriele d'Annunzio, i libri proibiti e i rapporti Stato-Chiesa all'ombra del Concordato, Roma, Edizioni di storia e letteratura, 2016.

${ }^{67}$ Barraband \& Camus, ‘Le combat culturel des traditionalistes catholiques', cit.
} 
inoltre, è particolarmente problematica: essendo Cristo una persona divina vera e simbolica allo stesso tempo, i credenti associati a Civitas e a AGRIFF non accettano che se ne faccia un personaggio, perché ogni rappresentazione di Cristo è Cristo. In ultima analisi il processo per blasfemia dimostra come i cattolici integralisti rifiutino la pluralità della società postreligiosa in generale, e non soltanto quella della finzione. ${ }^{68}$

\section{Il teatro tra spazio pubblico e privato}

Vengono quindi a scontrarsi al Theâtre de la Ville, sullo stesso palcoscenico, due concezioni diametralmente opposte dell'iconoclasmo dell'arte a soggetto religioso: una letterale e mimetica e una avanguardistica e antimimetica. Partendo dal presupposto che il teatro sia una sfera pubblica che insiste su un incontro pienamente democratico e apertamente partecipatorio, Sandra D'Urso nella sua analisi dell'occupazione del teatro a Parigi considera l'operazione di Castellucci problematica sotto vari aspetti, visto che in questo caso il teatro come agorà del dissenso civile ha dato luogo all'intervento della polizia. ${ }^{69}$ Se l'avanguardia, con il suo ricorso a corpi e soggetti non conformi alle aspettative borghesi, ha sempre preteso di costituire un 'contropubblico' in contrasto con le configurazioni dominanti del 'pubblico' borghese ${ }^{70}$ in questo caso invece della sua resistenza ha messo in atto politiche piuttosto regressive.

Ciò porta la studiosa a domandarsi se l'avanguardismo non sia altrettanto costituito nello spazio civile borghese, con tutte le sue presunzioni ed esclusività, nello stesso modo in cui lo sono i cristiani bianchi e conservatori che occupano il palcoscenico. ${ }^{71}$ Inoltre, la semiotica intenzionalmente secolare dello sguardo di Cristo proposta dal regista e inserita nel metodo illuminista di vedere e interpetare il mondo, non contempla la semiotica letterale adottata invece dai cristiani ortodossi, per cui ogni violenza all'immagine di Cristo sulla scena è percepita come un attacco alla persona del divino. ${ }^{72}$ D'Urso quindi si chiede se l'appello che Castellucci rivolge al pubblico, a interagire con il simbolo cristiano del volto, non si sia trasmutato in un evento politico che si è conformato, anziché resistere, al potere dominante della governamentalità dei corpi nel senso di biopotere datoci da Foucault. ${ }^{73}$ C'è inoltre, come osserva Christopher Balme, una contraddizione fondamentale nella definizione del teatro come spazio pubblico, inteso dai manifestanti come soggetto alle leggi contro la blasfemia, e invece dagli spettatori del teatro e dalla politica come affrancato dalla censura. Mentre i difensori della libertà artistica godono della protezione dello stato e quindi sono in diritto di 'privatizzare' lo spazio teatrale, i nuovi 'indignati' possono ricorrere solo a un'opposizione 'affettiva', sostenuta da un considerevole impegno fisico e mediatico, spostando così il loro raggio d'azione paradossalmente all'internet, altro foro pubblico libero dalla censura. ${ }^{74}$

Come già messo in evidenza, c'è da precisare che la reazione del mondo religioso non è stata uniforme. Si potrebbe anzi dire che proprio le proteste ne abbiano mostrata la pluralità interna. Jeanne Favret-Saada a tal proposito fa una distinzione tra 'devoti', che non transigono sul loro concetto del sacro e 'credenti', che si rifanno a una pluralità

\footnotetext{
${ }^{68}$ A. Arzoumanov, M. Barraband \& M. Laforest, “"Mais c'est une fiction!” Mobilisation et démobilisation d'une notion controversée dans l'affaire Golgotha picnic', in: ConTextes. Revue de sociologie de la littérature, 26 (2020), https://doi.org/10.4000/contextes.8709 (14 maggio 2020).

${ }^{69}$ D'Urso, 'On the Theology of Romeo Castellucci's Theatre', cit., pp. 35-36.

70 Ivi, p. 37.

71 Ivi, p. 38.

72 Ivi, p. 42.

73 Ivi, p. 43.

74 Balme, 'The Affective Public Sphere', cit., p. 111.
} 
di opinioni, anche all'interno dei loro organi di stampa. ${ }^{75}$ Dato che la blasfemia in epoca moderna non viene più perseguita dalla legge di Stato, gli integralisti cercano di riformulare l'accusa facendo ricorso al termine di 'cristianofobia'. L'intento è quello di far rientrare il supposto reato all'interno delle aree legislative coperte dalle norme antirazziste e anti-discriminatorie. ${ }^{76}$ Il punto del contendere è, dunque, quello della libertà di espressione all'interno di una società multiculturale, motivo per cui i rappresentanti della Chiesa incitano invece i cattolici ad adeguarsi a quell' 'etica di cittadinanza' richiesta a tutti nella società democratica e postsecolare di cui parla Habermas.

Nella citata lettera del Vaticano firmato da Monsignor Wells, non solo veniva pronunciato un 'no' a quanto può offendere la fede, ma anche un 'no' a 'qualsiasi azione di risposta che esca dai limiti della compostezza'. Invece, il Comitato S. Carlo Borromeo, il più agguerrito nelle proteste contro lo spettacolo di Castellucci a Milano, ha affermato di essere sostenuto nella sua intransigenza dal clero - 'Non solo abbiamo l'appoggio di Sua Eminenza il Cardinale Scola, Arcivescovo di Milano, ma ora abbiamo anche quello della Santa Sede' - mentre i loro seguaci verrebbero ingiustamente tacciati di 'antisemitismo', 'ignoranza' e di 'non essere cattolici maturi'. È quanto viene riportato per introdurre un appello contro la richiesta dei manifestanti di cancellare lo spettacolo, il che equivarrebbe per i promotori della petizione (Massimo Marino, Attilio Scarpellini, Oliviero Ponte di Pino) a un atto di 'censura preventiva':

Non si tratta di scegliere tra chi dice di aver scritto il suo spettacolo come una preghiera e chi, senza averlo visto, lo accusa di essere blasfemo [...]. Si tratta semplicemente di garantire a Romeo Castellucci la prima ed essenziale libertà di ogni arte e di ogni artista: quella di essere compreso o frainteso con cognizione di causa, di essere giudicato secondo la sua opera e non secondo il pregiudizio di un manipolo di fondamentalisti che agita la fede in Cristo come una clava identitaria. ${ }^{77}$

Nel 2018 lo spettacolo ha destato di nuovo scalpore al suo approdo a Le Mans, questa volta risultando in ciò che è stato percepito come un'effettiva "censura" preventiva, che ha messo in rilievo altre dimensioni dell'opera: la sua dimensione etico-pedagogica e l'uso di attori bambini nelle performance di Castellucci. ${ }^{78}$ Sandrine Blanchard su Le Monde riassume le reazioni all'accaduto, quella della ministra della cultura Françoise Nyssen che, interpellata, ha preso le distanze su Twitter - 'Je rappelle mon attachment profond à la liberté de création' -, e la denuncia da parte della SACD, la 'Société des auteurs et

\footnotetext{
$75 \mathrm{~J}$. Favret-Saada, 'Les jugements de blasphème et la politique des Etats démocratiques. Conférence au Salon du Livre en sciences humaines', novembre 2012, Paris, France, https://halshs.archives-ouvertes.fr/halshs01188431 (14 maggio 2020). Si veda per esempio l'oggettivo riassunto delle opinioni contrastanti su La Croix, 'Les pièces de théâtre de Roméo Castellucci et de Rodrigo Garcia continuent de susciter des réactions', 18 novembre 2011, https://www.la-croix.com/print/article/737463 (14 maggio 2020). Su Corrispondenza Romana, l'organo dei cattolici tradizionalisti in Italia, si legge invece a proposito delle manifestazioni a Milano: 'Lode dunque a tutti i buoni cattolici, a partire da mons. Negri e il cardinal Martino. Onta a quei "cattolici senza Cristo", che in un modo o nell'altro, hanno difeso la bestemmia in compagnia dell'armata laica: "Repubblica" (sempre in prima linea), "Il Corriere" (con Battista), "La Stampa”, “L’Unità", etc.'. F. Cannone, 'Oltraggi blasfemi: Romeo Castellucci secondo il Catechismo', 24 gennaio 2012, https://www.corrispondenzaromana.it/romeo-castellucci-secondo-il-catechismo/ (14 maggio 2020).

${ }^{76}$ Favret-Saada, 'Les jugements de blaspheme', cit.

${ }^{77}$ A. Bandettini, 'Il Vaticano vs Castellucci: ma la verità dov'è finita?', Post teatro - Blog - Repubblica.it, 19 gennaio 2012, http://bandettini.blogautore.repubblica.it/2012/01/19/il-vaticano-vs-castellucci-ma-laverita-dove-finita/ (14 maggio 2020).

${ }^{78} \mathrm{Si}$ veda M.B. Lafrance, 'Quand le réel entre en scène: la figure de l'enfant chez Castellucci', in: Jeu. Revue de théâtre, 142, 1 (2012), pp. 90-97.
} 
compositeurs dramatiques', che considera la misura della prefettura della Sarthe come 'un acte de censure au nom du prétexte fallacieux de la protection de mineurs et de la jeunesse'. A queste si aggiunge la comunicazione di Emmanuel Macron, alla Conferenza Episcopale Francese, in cui il Presidente della Repubblica osserva che 'Le lien entre l'Église et l'État s'est abîmé, il nous incombe de le réparer'. ${ }^{79}$ La prefettura, interrogata da Le Monde, assicura che non si è trattato di voler censurare una parte dello spettacolo, ma di voler salvaguardare i minori come previsto dalla normale procedura di consultazione delle istanze che tutelano il lavoro infantile. Viene negato inoltre un possibile legame con le proteste dei manifestanti, tra i quali un prete, che hanno esposto davanti al teatro degli striscioni nel tentativo di opporsi allo spettacolo. ${ }^{80}$ Castellucci nella lettera messa in rete sul sito del teatro les Quinconces-L'Espal dice di essere costernato dalla decisione prefettorale:

La moralité évoquée ici est ce qu'on appelle le sens commun: une caricature de la véritable éthique, une offense à l'intelligence critique des adultes et des enfants. L'art est une éthique contenue dans une esthétique et cela n'a rien à voir avec le moralisme. La Préfecture a certes le devoir d'œuvrer pour le bien de la société et de la préserver des dangers mais, dans le cas présent, ce type de réponse me semble mieux convenir à un régime théocratique qu'à une République fondée sur la liberté d'expression. ${ }^{81}$

Questa volta l'artista legittima il suo operato contrapponendo il suo impegno attivo a coinvolgere i bambini nella genesi dell'opera e nel ruolo che essi stessi svolgono come attori e interpreti nella seconda parte della performance, all'astratta morale a cui fanno riferimento i rappresentanti della Repubblica:

Pour monter cette scène, dans chaque ville nous organisons régulièrement des rencontres préparatoires avec les enfants, afin de leur faire comprendre "l'homéopathie" de ce geste violent qui appelle des sentiments inverses. ${ }^{82}$

Il regista trova sostegno in Arnaud Rykner, professore alla Sorbonne Université, la cui lettera del 12 aprile al prefetto della Sarthe è stata condivisa e sostenuta dalla rivista delle arti della scena L'Insensé, in cui accusa lo stato democratico per essersi fatto portavoce di un gruppo di integralisti cattolici che non rappresentano la maggioranza della popolazione francese. ${ }^{83}$ Tra le recensioni in rete anche quella di una spettatrice teologa e cristiana che sottolinea la dimensione spirituale dello spettacolo: 'J'en suis sortie bouleversée devant une telle qualité d'approche, une telle profondeur spirituelle. C'est d'une justesse et d'une finesse théologiques qui m'a sidérée'. ${ }^{84}$

\footnotetext{
79 S. Capron, 'Romeo Castellucci censuré au Mans: le Préfet fait supprimer une scène du Concept du visage du fils de Dieu', Sceneweb.fr, 13 aprile 2018, https://sceneweb.fr/romeo-castellucci-censure-au-mans-leprefet-fait-supprimer-une-scene-du-concept-du-visage-du-fils-de-dieu/ (14 maggio 2020).

${ }^{80} \mathrm{~S}$. Blanchard, 'Retour sur une pièce censurée de Castellucci', Le Monde, 19 aprile 2018.

${ }^{81}$ La lettera è riprodotta in S. Capron, 'Romeo Castellucci censuré au Mans', cit.

82 Ibidem.

83 'Castellucci Censuré. Lettre D’Arnaud Rykner Au Préfet De La Sarthe', L'Insensé, 13 aprile 2018, http://www.insense-scenes.net/?p=1605 (14 maggio 2020).

${ }^{84}$ M. -C. Bernard, 'A propos du spectacle de R.Castellucci. Réaction d'une théologienne', Le Maine Libre, 12 aprile 2018, riprodotto su http://www.mariechristinebernard.org/2018/04/12/concept-visage-fils-de-dieude-r-castellucci/ (14 maggio 2020).
} 


\section{Conclusioni: verso un'arte dello scandalo postsecolare}

In conclusione si potrebbe dire che la libertà artistica si esprima proprio attraverso le controversie che l'opera riesce a suscitare. Queste riguardano il postsecolare nella sua dimensione socioteologica e politica di una società democratica e multiculturale all'interno della quale il secolare e il religioso hanno perso la loro rispettiva normatività 'naturale', come ha dimostrato Parmaksı, e in cui 'religione' e 'spiritualità' sono diventati dei concetti non più necessariamente coincidenti..$^{85}$ La critica postreligiosa del postsecolare riguarda anche la dimensione filosofica-estetica di una tradizione avanguardistica che si rifà alla distruzione antimimetica con fini etici e che viene legittimata dalla libertà di espressione garantita dallo stato democratico. Tra le due parti contendenti, Castellucci e gli intellettuali laici da una parte e i cattolici tradizionalisti dall'altra, le opposizioni all'interno della dialettica tra 'pubblico' e 'contropubblico' nella terminologia di D'Urso si riconfermano perché radicate in semiotiche antimimetiche e mimetiche diametralmente opposte. Dall'altro lato invece, vista la non contraddizione inerente all'opera di Castellucci tra decostruzione e ricostruzione di significato, l'opera può parlare anche a un pubblico postsecolare, non più classificabile in una delle due categorie del secolare o del religioso, che potrebbe essere il destinatario ideale di un teatro dello 'scandalo' all'insegna dell' 'iconoclash'.

\section{Parole chiave \\ Postsecolare, iconoclasmo, blasfemia, libertà di espressione, Castellucci}

Monica Jansen insegna Letteratura e Cultura Italiana presso l'Università di Utrecht. I suoi interessi di ricerca vertono sulla letteratura e cultura italiana contemporanea, sul modernismo, postmodernismo e la memoria culturale della precarietà e della religione. Le sue pubblicazioni includono: Il dibattito sul postmoderno in Italia: In bilico tra dialettica e ambiguità (2002); diverse curatele tra cui le più recenti The History of Futurism: The Precursors, Protagonists, and Legacies (con Geert Buelens e Harald Hendrix, 2012), Le culture del precariato: Pensiero, azione, narrazione (con Silvia Contarini e Stefania Ricciardi, 2015), Televisionismo: narrazioni televisive della storia italiana negli anni della seconda Repubblica (con Maria Bonaria Urban, 2015), e Viaggi minimi e luoghi qualsiasi. In cammino tra cinema, letteratura e arti visive nell'Italia contemporanea (con Inge Lanslots e Marina Spunta, 2020). Codirige la collana Moving Texts/Testi Mobili (PIE Peter Lang), fa parte della redazione di Journal of Italian Cinema and Media Studies (JICMS), ed è caporedattore di Annali d'Italianistica.

Utrecht University

Dept of Languages, Literature and Communication - Italian Language and Culture Trans 10 3512 JK Utrecht (Paesi Bassi)

m.m.jansen@uu.nl

\footnotetext{
${ }^{85}$ G. Giordan, 'Dalla religione alla spiritualità: una nuova legittimazione del sacro?', in: Quaderni di Sociologia, 35 (2004), pp. 105-117: 'La polarità tra "religione" e "spiritualità" si può collocare proprio in questa particolare situazione di progressivo sfaldamento della legittimità tradizionale riconosciuta all'istituzione e dell'affermarsi del dispositivo democratico che tutela la libertà dell'individuo. [...] Religione e spiritualità, quindi, non si elidono a vicenda, ma sarebbero due approcci diversi che possono convivere nella ricerca di senso dell'uomo contemporaneo'.
} 
SUMMARY

When 'scandal' becomes provocation

The postsecular and Romeo Castellucci's theatre of iconoclasm

Romeo Castellucci's performance On the Concept of the Face Regarding the Son of God has led to a series of violent protests coordinated by Catholic groups in France (Paris 2011, Le Mans 2018) and Italy (Milan 2012) and has been judged as being blasphemous and 'Christianophobic'. Castellucci in the media has defended his piece using biblical terms and advocating his artistic right to create 'scandal'. This contribution intends to investigate the limits between provocation and 'scandal' with the help of a work of art that adoperates consciously an 'iconoclash' (Latour 2002) montage between avant-garde transgression and the symbolic code of Catholicism. While the freedom of artistic expression enjoys the protection of the state, this 'natural' right is put into question by the postsecular 'return of religion' in the public sphere (Habermas 2006). The analysis of the Catholic protests against Castellucci's piece and the director's aesthetic and ethical goals, both show how the configuration of postsecular art becomes part of the 'denaturalization' of secular and religious normativity in postsecular society (Parmaksız 2018). 